\title{
A Comprehensive Survey on Real-Time Applications of WSN
}

\author{
Ahmad Ali ${ }^{1, *}$, Yu Ming ${ }^{1}$, Sagnik Chakraborty ${ }^{2}$ and Saima Iram ${ }^{2}$ \\ 1 School of Electronics and Information Engineering, Hebei University of Technology, Tianjin 300401, China; \\ yuming@hebut.edu.cn \\ 2 School of Energy and Environmental Engineering, Hebei University of Technology, Tianjin 300401, China; \\ sagnik11@yahoo.com (S.C.); iramsaima88@gmail.com (S.I.) \\ * Correspondence: 201441901019@stu.hebut.edu.cn; Tel.: +86-156-2025-5099
}

Received: 15 October 2017; Accepted: 3 November 2017; Published: 7 November 2017

\begin{abstract}
Nowadays, the investigation of the Wireless Sensor Network (WSN) has materialized its functional area ubiquitously such as environmental engineering, industrial and business applications, military, feedstock and habitat, agriculture sector, seismic detection, intelligent buildings, smart grids, and predictive maintenance, etc. Although some challenges still exist in the wireless sensor network, in spite of the shortcoming, it has been gaining significant attention among researchers and technologists due to its versatility and robustness. WSN is subject to a high potential technology that has been successfully implemented and tested in real-time scenarios, as well as deployed practically in various applications. In this paper, we have carried out an extensive survey in real-time applications of wireless sensor network deployment in a practical scenario such as the real-time intelligent monitoring of temperature, criminal activity in borders and surveillance on traffic monitoring, vehicular behavior on roads, water level and pressure, and remote monitoring of patients. The application of the Wireless Sensor Network in the assorted field of research areas has been widely deliberated. WSN is found to be the most effective solution in remote areas which are not yet explored due to its perilous nature and unreachable places. Here, in this study, we have cited the recent and updated research on the ubiquitous usage of WSN in diverse fields in an extensive and comprehensive approach.
\end{abstract}

Keywords: wireless sensor network (WSN); wireless body area network (WBAN); master node; sensor; data collection; real time

\section{Introduction}

With the recent breakthrough in the field of sensors, real-time application has gained prominent attention among technocrats and researchers. In order to eradicate the challenges of the sensors, technologists and researchers found a solution by deploying the real-time applications of Wireless sensor network (WSN). The real-time sensors will instantaneously sense, record, and send feedback to the end user for further processing of the obtained information. Specifically, the real-time application is concerned with the performance of critical applications that need bounded delay latency. Real-time wireless communication is an emerging application arena of WSNs which has a potential significant research direction. Real-time applications able to monitor, respond immediately to user input, or control an external environment. The external environment can be connected to the computer system via sensors, actuators, and input-output devices. Wireless sensor networks that enable the network bounded delay guarantee, which is essential for the end to end packet delivery, are termed as real-time WSN [1,2].

Wireless sensor network (WSN) is of the utmost importance in computer networking for the searching area and in information assortment. WSN finds its application in several areas, including monitoring and data storage. Due to rapid urbanization, the span of the applications of WSN has been increasing enormously. WSN has numerous sensors which are communicated via sending data from 
one sensor to another sensor in a wide area via packets. WSN is ubiquitously used in various fields such as military surveillance [3], the health care industry [4], and other industries [5]. The emergence of3rd millennium industries makes it interesting to research WSN. The actual implementation of WSN was started in 1950 and the US military used this technology. The first mature WSN was named the "Sound Surveillance System" (SOSUS). It was used to detect the threat of underwater submarines. Acoustic sensors (a type of sensor used to check the amplitude of the waves) are used for (SOSUS) and this technology is also being practiced nowadays [6]. Today, in every part of life,a sensor is used to detect sound, detect any kind of vibrations, check the water level, detect the temperature level [7], in smart houses [8], in smart phones, and most importantly, we use some kind of sensors in the human body [9]. The WSN consists of nodes, and through these nodes, sensors communicate with each other. The hierarchy of these nodes is like a star where every node connects to each other or like a mesh topology. Each node collects information from this sensor and communicates it to other nodes. A sensor node basically consists of a microcontroller, transceiver, external memory, power source, and one or more sensors. WSN consists of sensor nodes, a user, and an interconnected backbone. Figure 1 presents WSN Network architecture consisting of sensor nodes, which are used in environmental monitoring. In the context of wireless communication, the sensor nodes communicate with each other and send the processed data to the sink node. All the nodes send data to the sink node, which is further sent to users through the internet [10].

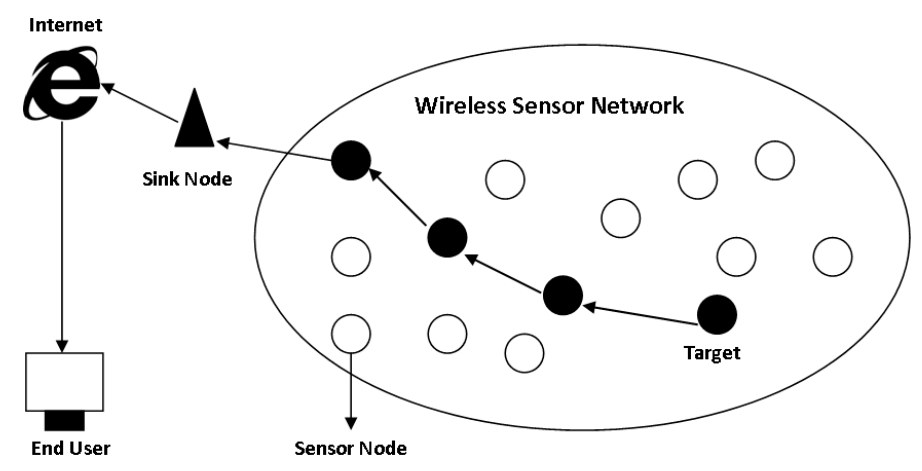

Figure 1. Wireless sensor network (WSN) System Architecture.

With the advancement in the sensor field like an embedded system, Micro-Electro-Mechanical System (MEMS), wireless communication, and disseminated processing, lots of changes have recently been brought about in WSN. An Intelligent system is capable of getting data and processes huge volumes of data, i.e., traffic management, weather monitoring, air quality management [11,12]. In this paper, we will discuss the real-time applications of WSN for monitoring the environment. These systems have replaced human efforts following an efficient method and could be able to gain functional requirements.

\section{Framework/Layout of the Study}

A real-time WSN is ubiquitously present in every aspect of daily life of human beings. Due to rapid development in society, there is an unmet need to make it smarter and more intelligent. Technologists and researchers are continuously pursuing research on an intelligent system which will cater the technology in a very simple and easy way for the common person. Several researchers have already put forward their review in WSN. In this paper, we have attempted to provide an extensive range of applications and implementations in the areas where it has been deployed, citing the examples. Real-time sensors have been implemented ubiquitously in society from agricultural farming to military surveillance. These examples have been cited with references. In this paper, we have segmented different sections with real-time sensors deployed in the city or province. Table 1 , starts with environmental monitoring; where air pollutant monitoring has been evaluated by the usage of 
sensors. From these sensors, the current status of the environment can be understood and further remedial measures can be implemented by the regulatory authorities of the cities or province. Table 2, is concerned with Green House Monitoring, which is pertaining to agricultural farming; via these sensors, the developmental stages of the fruit and vegetables could be monitored. Table 3, presents industrial applications of the sensor in a different industry such as a coffee making factory and gas filling industry. Table 4, summarizes the applications of WSN in livestock monitoring. Table 5, reports the application of WSN in habitat monitoring. Table 6, discusses the forest monitoring application; sometimes, unwanted fire is an outbreak in the forest and causes uncontrollable loss. Table 7, discusses the underwater sensor network; for water level, military surveillance, tracking, and tackling disasters prior to their occurrence. Table 8, elucidates healthcare monitoring via the usage of real-time sensors. A real-time sensor plays a significant role in the diagnostic division of the health care industry. Table 9, confers the application of a wearable sensor in the health care industry. Table 10, deals with Vehicular Ad Hoc Network (VANET) which basically communicates between the vehicles within the network for an intelligent transportation system. Table 11, discusses the intelligent vehicle system. Table 12, summarizes the application in military surveillance for security of the country.

\section{Types and Requirements of WSN}

WSN has many types of sensors depending on the situation such as underground WSN, underwater WSN, terrestrial WSN, multimedia WSN, and mobile WSN. Figure 2, represents the classifications of WSN.

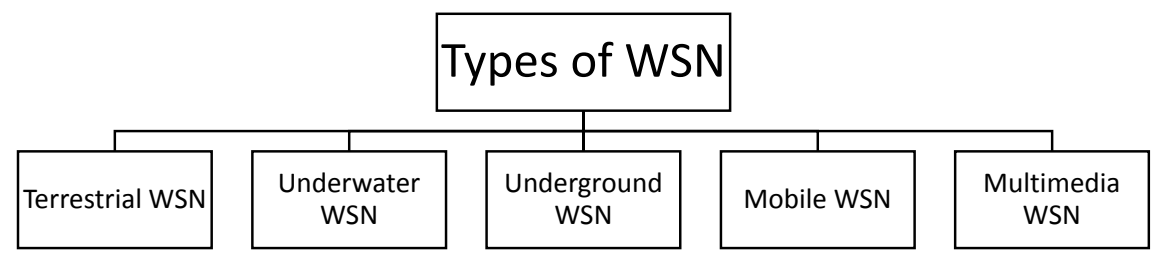

Figure 2. Classifications of WSN.

\subsection{Terrestrial Wireless Sensor Network}

Terrestrial WSNs consist of numerous tiny sensor nodes. These nodes are randomly deployed in a specific area from where an ad hoc network is used for communication between the nodes. These nodes can be organized by optimal placement, grid placement, or 2D and 3Dplacement models [13]. Nadeem et al. (2009) investigated this for applications of the terrestrial wireless sensor network in Radio Frequency/Free Space Optics (RF/FSO) systems, including open research issues and challenges. The drawbacks of terrestrial applications are the effect of weather such as rain and snow on an optical wireless communication link. The author has proposed a method to increase the lifetime performance of the network [14].

\subsection{Underground Wireless Sensor Network}

An underground WSN is a sensor node which is placed under the ground for collecting information on underground conditions. The drawback of WSN is its limited battery power because this is very difficult to recharge or change. The wireless communication's main limitation is the loss of signal at high potential. Therefore, in order to enhance the reliability of WSN, efficient routing algorithms have been carried out for solving the limitation of the WSN's accuracy during the routing process [15].

Zhe et al. (2011) proposed a mathematical framework in order to analyze the dynamic connectivity in the wireless underground sensor network (WUSN). Their aim was to monitor the changes in the environmental parameters such as the soil composition and the random soil moisture [16]. Idrees et al. (2016) developed a sensor node named Molenet to handle the wireless underground operation. The proposed system is part of a revitalization project in Cameroon. Their main goal was to keep the network operational for a long time and enhance the communication range [17]. 


\subsection{Underwater Wireless Sensor Network}

An underwater WSN includes very expensive sensor nodes, which are placed under the water for collecting data and information about underground ambiance. Some limitations or challenges of wireless communication underground include the delay of the network, limited bandwidth, and poor network signal. The aforesaid problem has been recognized by investigators, and in order to eradicate the problem, they proposed a solution via a suitable routing algorithm [18]. Davide et al. (2009) proposed an underwater wireless sensor network based on optical communication between nodes. Radio frequencies and acoustic waves are profoundly attenuated in water. To overcome this problem, optical communication can be considered. In order to increase the efficiency of the system, the authors designed a user interface for managing the optical Physical (PHY) layer implementation on a Digilent Spartan 3 Board [19]. Nithin et al. (2017) have assembled a review for prevalent approaches and issues relating to a certain parameter of research. They have presented a paper based on a survey of the underwater wireless sensor network with data aggregation to highlight its advantages and disadvantages. The authors implemented different clustering techniques for performing data aggregation as a result to demonstrate the impact of data aggregation [20].

\subsection{Mobile Wireless Sensor Network}

A mobile WSN has the advantage that power changes its position and automatically connects to the environment. Mobile sensors connect with computers and communicate. Mobile sensors are used for collecting data from a wide area or information from other nodes or sensors. The main limitations of mobile WSNs are the present status, coverage area, navigation, reposition, and maintenance. The aforementioned issues have been resolved via a dynamic routing algorithm [21]. Cho et al. (2011) have assembled a review of prevalent schemes for the mobile wireless sensor network, while keeping a certain parameter as the center of research. Various parameters include accepted classifications, potential attacks, performance metrics, and trust metrics on the mobile wireless sensor network [22].

\subsection{Multimedia Wireless Sensor Network}

A multimedia WSN consists of tiny sensor nodes that can sense, compute, actuate, and communicate. A variety of applications of the WSN include habitation monitoring, traffic management systems, and ecological monitoring. The multimedia WSN is a network of wirelessly interconnected devices that are able to ubiquitously retrieve video and audio transmission, still images, and scalar sensor data from the environment [23]. Akyildiz et al. (2006) analyzed the recent developments on multimedia WSNs and outlined the primary research challenges. Algorithms, protocols, and hardware for the enhancement of multimedia WSN sensors were surveyed and open analyses were conducted for the problems mentioned. The authors tend to classify ready-made hardware as an accessible research prototype for the multimedia WSN [24]. Muhammad et al. (2017) focused on some advanced well known multi channel assignment protocol in WSN. They have listed challenging issues and the classification of different channel selection policies, types of channel assignment, and methods of channel assignment [25].

Some Key Requirements of Wireless Sensor Networks

- Scalability: It will expand this network to add nodes as required. Its expansions should be easy to conduct.

- Reliability: Many methods are there for reducing the power usage of (WSN) nodes, which are resulting in an increase in the lifetime of the network and their consistency.

- Responsiveness: The response time is very quick because of its limitations.

- Mobility: Mobility is the basic feature of WSN. It is a wireless network so no wire is used for this network. That is why mobility is a key requirement of WSN.

- Power efficiency: It uses a sensor that results in low power consumption. 


\section{Applications of WSN}

The real time application of WSN is like a hot cake. WSN is ubiquitously used everywhere in modern society. It has been successfully implemented in diverse fields such as military, habitual, business, health, and industry applications. Furthermore, there are adequate scopes of research themes that have not yet been explored pertaining to this field. Some of the applications have been delineated below.

\subsection{Environment Monitoring}

There are various requirements of environmental monitoring applications, which include measuring the parameters of wind speed and direction. Figure 3, depicted the outline of environment monitoring system applications for WSN. Many of them are slow in changing behavior which allows one to sample them efficiently from one to five minutes as they react. Yet, a fascinating phenomenon of rockslides has been difficult to predict in such a case and the developments should be long lasting in order to capture them. The following technical requirements are needed for the environment checking system [26]. Table 1 , represent the examples of real-time environment monitoring system applications that utilize WSNs.

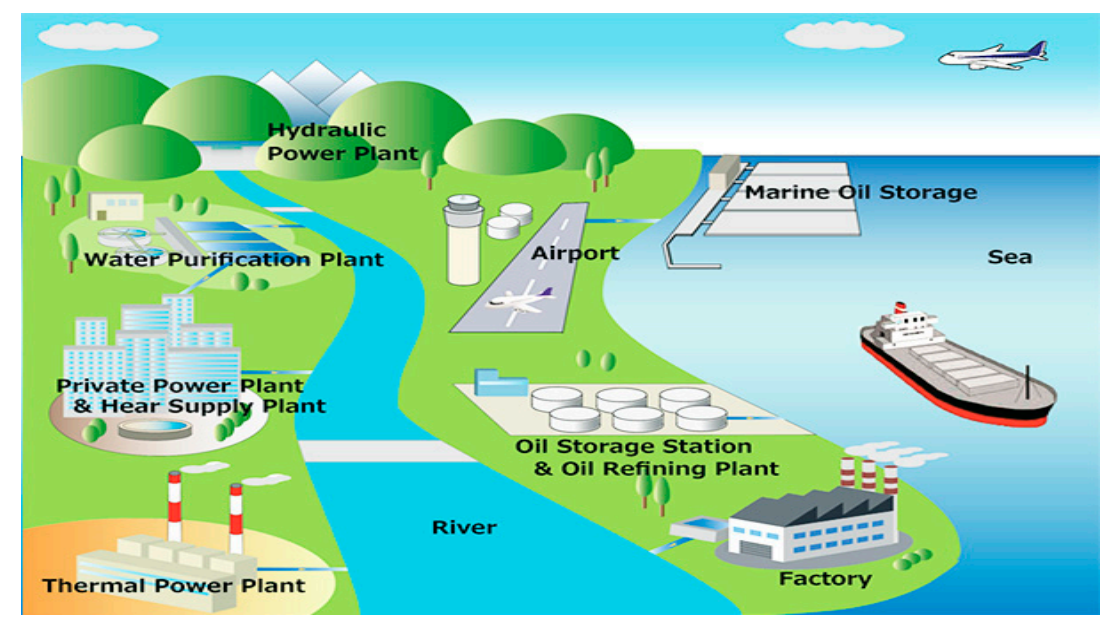

Figure 3. Environment monitoring system.

\subsubsection{Autonomy}

The power of batteries should be large enough to support a weather station throughout the whole operation. The condition of switching on the radio must not be entertained all the time. Usage of the solar system can be a good option.

\subsubsection{Reliability}

To prevent an unforeseen crash, the network should perform very simple and expected operations. The end user may not have enough knowledge and the interested areas are isolated most of the time. During the ruthless situation of weather (like very cold weather and a lot of rain in), the packet could be lost so it becomes harder to achieve reliability.

\subsubsection{Robustness}

The concerning network should be able to diagnose this problem, including the poor connectivity of radio transmission in the case of hardware failure or a bad weather situation like snow falling. It has been observed that humidity can lead to short-circuits, which may lead to restarting of the stations. Sometimes it is better to incorporate and remove the stations based on need. The new station could be added at any point of interest. It is not advisable to solely depend on earlier knowledge while redesigning the network. 
Boustani et al., 2011 investigated and employed the tracking system in Seattle, New York, for the recognition and improvisation of the waste management system. Boustani et al., deciphered the techniques for mobility of the waste from one point to another point via the Trash Track Project, which also provided insight regarding the tag performance and some attained traces. This technology also produced a concept and idea regarding the transformation and perception of the cities.

Mao et al., 2012 studied the real time surveillance for $\mathrm{CO}_{2}$ monitoring in a specific urban area network. They conducted their study via simulations resembling practical deployment in Wuxi, PR China.

Hasanfratz et al., 2014 studied the largest spatially resolved Ultrafine Particles (UFPs) data set in Zurich, Switzerland, which pertaining to air pollution measurements. The target pollutants are those such as nitrogen dioxide $\left(\mathrm{NO}_{2}\right)$, carbon monoxide $(\mathrm{CO})$, ozone $\left(\mathrm{O}_{3}\right)$, and Ultrafine Particles (UFPs), which are damaging to human health. These pollutants cause severe diseases such as respiratory problems, cardiovascular problems, etc. They collected the data for more than two years. The authors compared the accuracy models on a different time scale and found that there are significant changes obtained during their time frame. Furthermore, they developed a technique via modeling in which the previous data were incorporated with the metadata, and the result was shown to be quite significant. Additionally, after deployment of the model, they obtained a significant reduction in the root mean square values which inferred that the data is statistically reproducible.

Jiang et al., 2016 studied the deployment of sensors for air quality monitoring in Amsterdam, the Netherlands. The target compound was $\mathrm{NO}_{2}$. The authors delineated the mechanism based on a bottom-up approach for the citizen of the urban area. They compared an electrochemical sensor and a semiconductor sensor. Furthermore, they concluded that these highly sensitive electrochemical sensors exhibit an enhanced performance compared to a semiconductor sensor.

Table 1. Examples of prototyped applications for environmental monitoring. GSM: Global System for Mobile; UFPs: Ultrafine Particles.

\begin{tabular}{cccc}
\hline References & Application & Sensors & City Deployed in \\
\hline Boustani et al. (2011) [27] & Waste Removal & $\begin{array}{c}\text { Global System for Mobile (GSM) } \\
\text { Tracking Sensors }\end{array}$ & New York, Seattle \\
\hline Mao et al. (2012) [28] & City See $\mathrm{CO}_{2}$ Monitoring & $\mathrm{CO}_{2}$, Temperature, Light Sensors & Wuxi, China \\
\hline Hasanfratz et al. (2014) [29] & Air Pollution Maps & $\begin{array}{c}\mathrm{NO}_{2}, \mathrm{CO}, \mathrm{O}_{3}, \text { Ultrafine Particles } \\
\text { (UFPs), Temperature, Humdity }\end{array}$ & Zurich, Switerzerland \\
\hline Jiang et al. (2016) [30] & Air Quality & $\mathrm{NO}_{2}$ Sensors & Amsterdam, The Netherlands \\
\hline
\end{tabular}

\subsection{Green Houses}

The main objective was to save heat energy in greenhouses by smoothly measuring the temperature with high resolution horizontal data, as well as vertical data, for achieving appropriate and valid results with the help of previously mentioned modules that were employed in collaboration with an agricultural chamber [31]. Figure 4, represents the real-time usage of WSNs in precision agriculture. Table 2, depicts the examples of real-time greenhouse monitoring system applications that utilize WSNs.

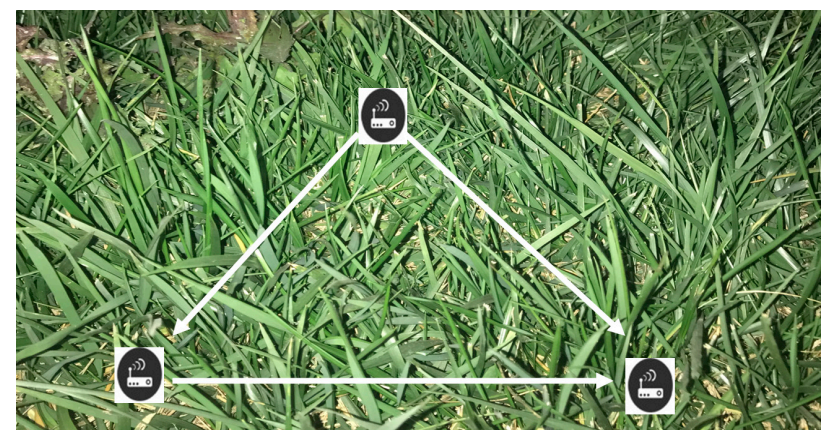

Figure 4. The uses of WSNs in precision agriculture [32]. 
Pahuja et al., 2013 employed a system for greenhouse climate control in Punjab, India. They developed a system which monitors and analyzes the ambient atmospheric parameters for the development of plants. For the improvisation and enhancement of the techniques, they incorporated and integrated a system which automatically monitors, analyzes, and resolves the issues pertaining to their problems.

Liu et al., 2016 developed an intelligent monitoring system for their farming of grapes in their greenhouse. They incorporated the parameters which have a significant role in the development of the grapes. The whole system or mechanism could be monitored via online or real time. Additionally, sensors recorded each and every single developmental stage via a video and image capturing technique. The data set was generated via the real-time sensors, and created an active database for further investigation and analysis.

Table 2. Examples of prototyped applications for greenhouse monitoring.

\begin{tabular}{cccc}
\hline References & Application & Sensors & City Deployed in \\
\hline Pahuja et al. (2013) [33] & Climate Control & $\begin{array}{c}\text { Vapor pressure deficit (VPD), } \\
\text { Humidity, Temperature }\end{array}$ & Punjab, India \\
\hline Liu et al. (2016) [34] & $\begin{array}{c}\text { Intelligent Environment } \\
\text { Control System }\end{array}$ & $\begin{array}{c}\text { Multiple Sensors, illumination, } \\
\text { Soil Moisture, } \text { CO }_{2}\end{array}$ & Jiangsu, China \\
\hline
\end{tabular}

\subsection{Industrial and Business Uses}

This is very helpful in an industrial application. These sensors are also used for monitoring, controlling, and processing the data, such as pressure, vibration, temperature, and viscosity. Sensors collect the data or information sent to the control system management. They also play a pivotal role in establishing the business process. A better routing algorithm provides us with a long time, an easy to use system, and low cost devices which are very helpful in business applications [35]. The WSN uses the building decision support systems, avoiding various problems in the real world. The decision support system is also being employed increasing widely in agriculture to solve the most complex problems of agriculture related to the monitoring of land. This approach will be valid for helping the farmers make the right decisions. The rapid and progressive development of WSN applications in precision agriculture makes it possible to enhance the efficiency and productivity in various agriculture production systems. Additionally, farmers get an insight and detailed information pertaining to their fields and find their best solution via the usage of WSN. Table 3, represents the examples of real-time applications for industrial and business uses that utilize WSNs.

$\mathrm{Ni}$ et al., 2009 investigated the gas meter sensor system. They developed a network hierarchy, such as receiving, transmitting, auto-reconfiguration, scheduling, and deep sleeping strict requirement of energy utilization.

Chen et al., 2011 studied the reduction of air conditioning energy utilization. They gave an insight into the mechanism of how to reduce the utilization by predicting a mean vote and computational fluid dynamics approach.

Valverde et al., 2012 investigated a coffee factory in Madrid, Spain. They installed the sensors in the industry and it is a very daunting task to deploy sensors. They installed them everywhere in the production unit from raw coffee to the final product. They installed the hardware system along with the software for better sensing the pollutants in the industry. 
Table 3. Examples of prototyped applications for industrial and business uses.

\begin{tabular}{cccc}
\hline References & Application & Sensors & City Deployed in \\
\hline Ni et al. (2009) [36] & Gas Meter & Pressure Sensors & Taipei, Taiwan \\
\hline Chen et al. (2011) [37] & Convenience Store & $\begin{array}{c}\text { Temperature, Humidity, } \\
\text { Micro-Electro-Mechanical Systems } \\
\text { (MEMS), flow Sensors }\end{array}$ & Taipai, Taiwan \\
\hline Valverde et al. (2012) [38] & Monitoring for Coffee Factory & $\begin{array}{c}\mathrm{NO}, \mathrm{SO}_{2}, \mathrm{CO}, \mathrm{pH} \\
\text { And Water Temperature }\end{array}$ & Madrid, Spain \\
\hline
\end{tabular}

\subsection{In the Livestock}

A wireless detection system helps to keep livestock healthy with a minimum use of resources. The system detects the temperature and $\mathrm{pH}$ level inside the rumen of cows. The data are wirelessly transmitted to an external receiver node via an encapsulated measuring probe referred to as bolas. The finding of this application is developing a wireless rumen checking system for the detection of sub acute rumen acidosis by the quasi-continuous measurement of transmission/indication of the $\mathrm{pH}$-value of the rumen of dairy cows $[39,40]$. Table 4 , represent the examples of real-time applications for livestock that utilize WSNs.

Wark et al., 2007deployed the sensors among cattle for understanding their mood, stress, hunger, etc., in Queensland, Australia. They deployed Radio-Frequency Identification (RFID) tags on their ears for obtaining all of the information in real time.

Nkwari et al., 2014 deployed the sensors to eradicate the theft of stock for cattle in Johannesburg, South Africa. They incorporated the Continuous Time Markov Process for the movement of cows. The sensor provides the speed of mobility of the cow which is used as a reference for any movement of the animal. They successfully stopped the theft of animal stock in farms, especially in South Africa and Africa.

Wamuyu et al., 2017 investigated the tracking of animal's movement for the prevention of theft in Nairobi, Kenya. The authors studied how to recognize and track the preferred animal's location and send the positioning data in a periodic manner to a database. Data from the database was sent on a demand basis via the internet and short messaging system or texts. They designed a sensor module which consists of Worldwide Interoperability for the Microwave Access (WiMAX) gateway, WiMAX base stations, and data collection centers.

Table 4. Examples of prototyped applications for livestock. WiMAX: Worldwide Interoperability for the Microwave Access.

\begin{tabular}{cccc}
\hline References & Application & Sensors & City Deployed in \\
\hline Wark et al. (2007) [41] & Automated Cattle Monitoring & Motion Sensors, ECH $\mathrm{E}_{2} \mathrm{O}$ & Queensland, Australia \\
\hline Nkwari et al. (2014) [42] & Prevent Cattle Rustling & Markov Process, GSM, Wi-Fi & $\begin{array}{c}\text { Johannesburg, } \\
\text { South Africa }\end{array}$ \\
\hline Wamuyu et al. (2017) [43] & Cattle Recovery System & $\begin{array}{c}\text { Mobile Embedded Sensors, } \\
\text { Worldwide Interoperability for } \\
\text { the Microwave Access (WiMAX) }\end{array}$ & Nairobi, Kenya \\
\hline
\end{tabular}

\subsection{Habitat Monitoring}

Researchers in the life sciences are becoming increasingly concerned about the potential impacts of human presence when observing plants and animals in field conditions. In life sciences, huge numbers of researchers are based on the impact of the human presence of observing animals and plants in field conditions [44]. WSN is also employed in monitoring the habitat. Of late, it has been observed that due to the activity of trespassers in the habitat area, a disturbance in the breeding of the plants or sometimes in the animal husbandry area has been created. The unwanted stress has resulted in a low breeding rate within the specific species that has been monitored by the WSN [45]. Table 5, represents the examples of real-time applications for habitat monitoring that utilize WSNs. 
Mainwaring et al., 2002 investigated the application of the real-time sensors to visualize and monitor seabird nesting and its ambiance in California, USA. They deployed the sensors on a small Island off the coast of Maine for gaining an insight into live data on the web. They identified key areas of the research such as data sampling, communications, network rescheduling, and health monitoring.

Table 5. Examples of prototyped applications for habitat monitoring.

\begin{tabular}{cccc}
\hline References & Application & Sensors & City Deployed in \\
\hline Mainwaring et al. (2002) [46] & $\begin{array}{c}\text { Monitoring Sea Bird } \\
\text { Nesting Environment }\end{array}$ & $\begin{array}{c}\text { MICA Weather Board, Temperature, barometric } \\
\text { pressure, passive infrared sensors }\end{array}$ & California, USA \\
\hline Naumowicz et al. (2010) [47] & $\begin{array}{c}\text { Monitor Sea Birds on } \\
\text { Skomer Island }\end{array}$ & $\begin{array}{c}\text { Temperature, Humidity, Movement, } \\
\text { Radio-Frequency Identification (RFID), Weight }\end{array}$ & Wales, UK \\
\hline
\end{tabular}

\subsection{Forest Monitoring}

The value of the forest and its importance on earth plays a vital role in human life and an unparalleled role in maintaining the biogeochemical cycle of the atmosphere. Due to urbanization, afforestation is of the utmost importance for society. Additionally, for surveillance of the forest, monitoring is required. For maintaining safety, WSNs are usually employed in the aforesaid area. Figure 5, represents real-time sensor deployment for forest monitoring.

Monitoring the micro-climate in the woods is becoming increasingly important, given the predicted climate change. In-depth knowledge of individuals about the physical, chemical, and biological status of soil in the root system of trees makes a significant contribution to the intensive monitoring of forest ecosystems. These long-term studies are a prerequisite for the derivation of measures for forest management. Forest operation remains unaffected by the wireless transmission of sensor readings. Instead of that, wireless transmission ensures robustness, as compared to a wired installation of sensors $[48,49]$. Table 6 , depicts the examples of the real-time applications for forest monitoring that utilize WSNs.

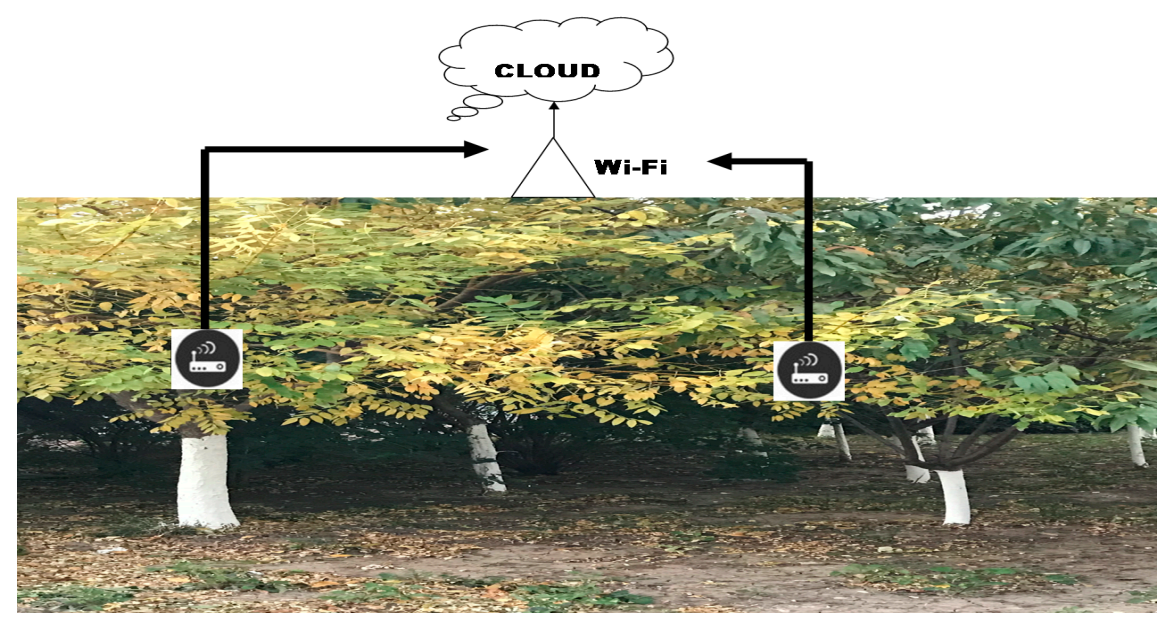

Figure 5. Sensor deployment for forest monitoring [50].

Son et al., 2006 studied and developed a Forest Fire Surveillance System (FSSS) in Daegu, Korea. Based on the literature, it was reported that no aforesaid surveillance was present. Son et al., 2006 developed the FSSS, which they incorporated and integrated into the system for surveillance in the forest. The FSSS consists of WSN, middleware, and a real-time approach. The system will detect the physicochemical parameters of the environment such as temperature, humidity, and perceived smoke. When any kind of heat and smoke is detected by the sensor, it will automatically generate an alarm regarding the possibility of a forest fire. 
Hafeeda et al., 2007 investigated the real-time application of sensors for the detection of the Forest Fire detection index in Columbia, Canada. The authors developed a data aggregation scheme-based Fire Weather Index (FWI) system. Additionally, the obtained data of interest were validated with other models for the system's reproducibility. The K coverage algorithm works as a function of the early detection of fire in the forest vicinity. The developed algorithm is simple, robust, and can be deployed anywhere.

Table 6. Examples of prototyped applications for forest monitoring.

\begin{tabular}{cccc}
\hline References & Application & Sensors & City Deployed in \\
\hline Son et al. (2006) [51] & $\begin{array}{c}\text { FFSS-Forest Fire } \\
\text { Surveillance System }\end{array}$ & $\begin{array}{c}\text { Temperature, Humidity, } \\
\text { Light Sensors }\end{array}$ & Daegu, Korea \\
\hline Hefeeda et al. (2007) [52] & FWI-Fire Weather Index & $\begin{array}{c}\text { Temperature, Humidity, } \\
\text { Moisture, Temperature }\end{array}$ & Columbia, Canada \\
\hline
\end{tabular}

\subsection{Underwater Sensor Network}

Wireless sensor networks (WSNs) are an emerging technology today, and are made up of a large number of separated tiny embedded sensor nodes that are used in monitoring and sending data from their surroundings [53]. The research in the underwater environment is facing many challenges in localization, node mobility, end to end delay, dynamic topology, energy consumption, and effective communication. In underwater wireless sensor networks, routing is different from the terrestrial wireless sensor networks due to a limited bandwidth, node mobility, and end to end delay in data packet transmission. Existing routing protocols have been analyzed which investigate the optimization performance of network services, node mobility, end to end delay, and energy draining of sensor nodes. Large numbers of routing protocol have been developed for those that are working in Underwater Wireless Sensor Network (UWSN). These protocols evaluate the performance efficiency with respect to an end to end delay, node mobility, network throughput, and energy consumption, etc. [54]. Figure 6, shows the real-time example of underwater sensor network implemented in Aquarium of Zaragoza. The UWSN consists of four different numbers of sensor nodes. Nodes that are anchored to the sea bottom have randomly floated with the water current. Anchored nodes sense data within range environments and route the sensed data toward the surface sink in multi-hop fashion or through autonomous underwater vehicles (AUVs). The autonomous underwater vehicles (AUVs), unmanned underwater vehicles (UUVs) move in water in a fixed pattern to communicate with sensor nodes through a short range and high rate data link. AUVs near the base station negotiate to the sink node to forward the collected data. In UWSN, link failure is a burning issue due to node mobility. Node mobility will create holes in the network causing an increase in the end to end delay in data transmission. The efficient underwater communication poses significant problems due to the intrinsic absorption nature of water. The acoustic signal also faces many problems due to a high error rate, low available bandwidth, node mobility, lower propagation speed, and high end to end delay. In recent years, the wireless sensor network applications have spanned into different fields for use in weather monitoring; water pollution monitoring such as biological, chemical, nuclear, and micro-organism tracking; and disaster prevention. UWSN is also widely used in underwater oil and gas pipeline corrosion detection and military security applications. In order to design an efficient UWSN routing protocol, researchers face many challenges which attenuate the medium, such as node mobility, end-to-end propagation delay, and energy saving [55]. Table 7, depicts the example of the real-time applications for underwater sensor networks. 


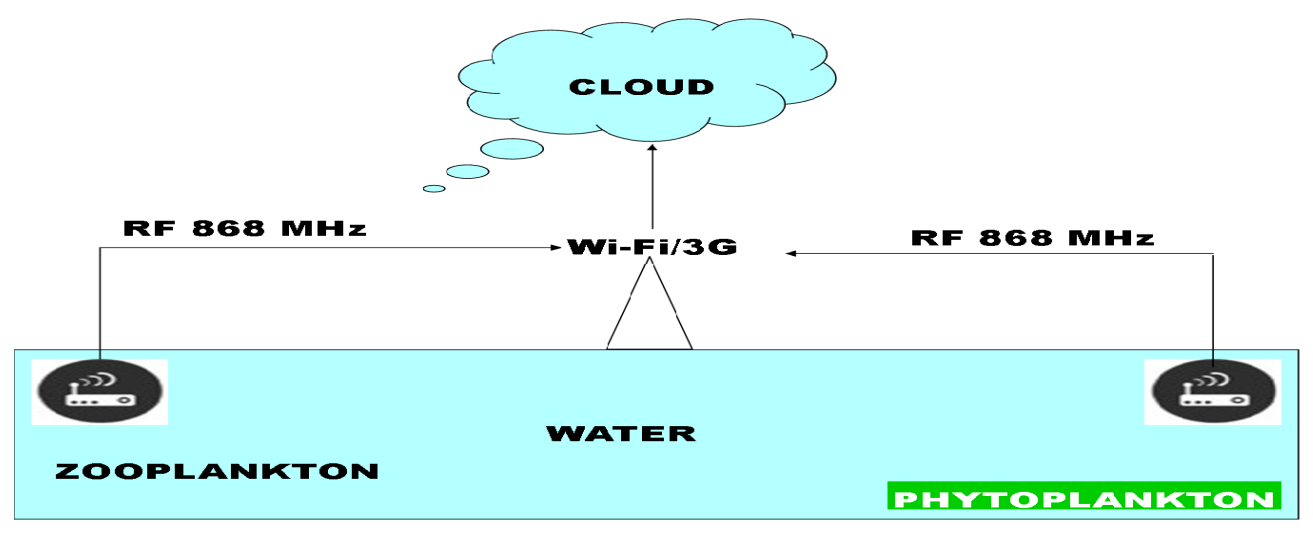

Figure 6. Aquarium of Zaragoza Solution Diagram [56]. RF: Radio Frequency.

Gray et al., 2011 investigated and deployed real-time sensors for flood retort development in Portsmouth, England. The authors delineated the semantic sensor web architecture which involved several heterogeneous databases and data sets. The architecture provides an insight into the mechanism for discovering the data sets, defining incorporation-integrated views, real time streaming, visualization, and communication with the obtained information. The authors have established the application of semantic sensor web pertaining to the flood retort development, web architecture. The architecture will employ the obtained information from the sensor network monitoring nearby the coast of England.

Whittle et al., 2013 investigated and developed a real-time sensor with the intelligent sensor network in Singapore. They monitored water quality parameters, acoustics, and the hydraulic retention state. The authors developed the tool for monitoring, detecting, and unraveling the abnormal behavior phenomena for long term trends.

Kim et al., 2017 investigated the real-time application of WSN in Korea by developing the Underwater Wireless Sensor Network (UWSN) named SOUNET. The authors enhanced the network connectivity, packet delivery ratio (PDR), and energy utilization. Additionally, they compared their study with the commercially procured modem to validate the efficiency of SOUNET in a practical or real environment.

Table 7. Examples of prototyped applications for underwater situations.

\begin{tabular}{cccc}
\hline References & Application & Sensors & City Deployed in \\
\hline Gray et al. (2011) [57] & $\begin{array}{c}\text { Environmental Decision } \\
\text { Support }\end{array}$ & Water Level Sensors & Portsmouth, UK \\
\hline Whittle et al. (2013) [58] & WaterWiSe@SG & $\begin{array}{c}\text { Temperature, Humidity, } \\
\text { Moisture, Temperature }\end{array}$ & Singapore \\
\hline Kim et al. (2017) [59] & SOUNET & $\begin{array}{c}\text { RF (radio frequency)antenna, } \\
\text { Underwater Modem }\end{array}$ & Mungyeong, Korea \\
\hline
\end{tabular}

\subsection{WSN and Health}

In health, WSN uses an advanced medical sensor to improve health applications. WSN is used to monitor diseases like Alzheimer's and heart attacks [60]. Table 8, depicts the examples of real-time applications for health monitoring system.

Ko et al., 2006 investigated a real-time sensor at hospital indoor ambiance and found that it was quite significant. They studied the blood oxygen level and heart rate of the patients admitted in the Emergency units of John Hopkins Hospital, USA. The authors collected the statistics of the network RF links, the performance of tree routing, and its reality in the network. The authors inferred that the real-time sensor has a potential application in the healthcare sector. 
Hii et al., 2011 investigated and incorporated the real-time sensors on smartphones and made them more user friendly in the health care system. They integrated the sensors on mobile phones via the usage of a barcode. The mechanism they delineated is the only comprehensive for the Electrocardiogram (ECG) and for the future scope of work they have suggested that the monitoring of the blood glucose level, blood pressure, and several kinds of diagnostics could be possible via the application of real-time sensors.

Kakria et al., 2015 investigated real-time sensors for the diagnosis of cardiac patients. The authors selected the age group 18-66 years, and among this age group, they selected some individuals. The authors introduced this application to cater for severe cardiac patients who are unable to attend a routine checkup. One of the main reasons behind this technology is to commercialize it for the benefit of those patients who are not financially sound. They have deployed these sensors under the supervision of experts. Furthermore, the system is developed in such a manner that any kind of crisis phase will be generated in terms of messages that will be automatically sent to the doctor.

Table 8. Examples of prototyped applications for health. ECG: Electrocardiogram; CDMA: Code Division Multiple Access.

\begin{tabular}{cccc}
\hline References & Application & Sensors & City Deployed in \\
\hline Ko et al. (2006) [61] & $\begin{array}{c}\text { Improved Efficiency } \\
\text { and Quality of Care }\end{array}$ & MiTags & John Hopkins Hospital, USA \\
\hline Hii et al. (2011) [62] & $\begin{array}{c}\text { Ubiquitous } \\
\text { Healthcare Solution }\end{array}$ & $\begin{array}{c}\text { Electrocardiogram (ECG) Sensor } \\
\text { Node, 3G, Code Division Multiple } \\
\text { Access (CDMA), GSM }\end{array}$ & Busen, Korea \\
\hline Kakria et al. (2015) [63] & $\begin{array}{c}\text { Real Time } \\
\text { monitoring System }\end{array}$ & $\begin{array}{c}\text { Wearable Biosensors, Android Device, } \\
\text { Smart Phone }\end{array}$ & Pathumthani, Thailand \\
\hline Yan et al. (2017) [64] & $\begin{array}{c}\text { Structural Health } \\
\text { MonitoringSystem }\end{array}$ & $\begin{array}{c}\text { Wireless Smart Aggregate sensor, RF } \\
\text { Module, Sampling Module, Gateway }\end{array}$ & Shenyang, China \\
\hline
\end{tabular}

\section{Wireless Body Area Network (WBAN)}

The usage of wireless devices has been increasing constantly; many modern technologies have been designed that are using wireless networks, producing decent results and making many challenging and demanding technologies accessible and easy to use. Special wireless devices for healthcare systems are called Wireless Body Area Networks (WBANs). WBANs introduced a dynamic arena of development and research into today's life. WBANs contributed their application ubiquitously. The literature suggests that WBANs will broaden their horizon in terms of a human being's activity. Pertaining to human health, WBAN systems give an insight into the physiological conditions of a human being. Generally, WBANs monitor the real time condition of health and send feedback to the end user or physicians via the connectivity. Mostly, they are used to monitor physiological parameters such as the heartbeat, temperature, stress level, and oxygen level. In spite of these physiological parameters, they also monitor and analyze the routine of exercise and the requirement of the human body. Figure 7, shows the overview of WBAN architecture. 


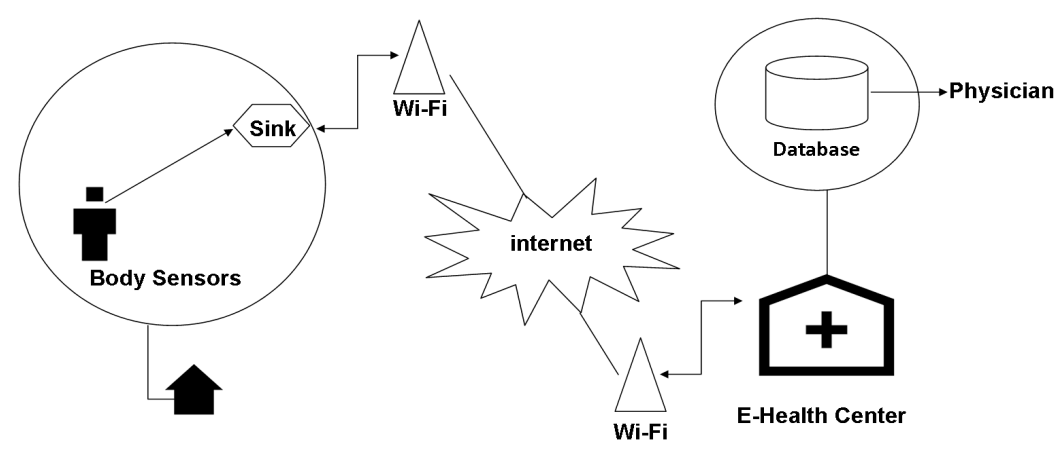

Figure 7. Wireless Body Area Network (WBAN) Architecture.

In this world, where the population is growing vastly but the resources are not sufficient, there should be some systems that satisfy imminent health care demands. The main purpose behind these low power wireless body area network devices is to efficiently utilize hospital facilities for those patients who need more care than those whose health is not critical, but still, the monitoring of their health is required and due to any reason they are not able to stay in the hospital for longer. All WBAN applications are based on three-tier architecture. All three tiers or levels are based on some devices, and these devices help WBANs to get and monitor data obtained from the human body and these levels are defined as follows $[65,66]$. Table 9, Shows the examples of real-time applications for wireless body area networks.

- Level 1: First level of WBAN architecture is called the body sensor unit that is based on one or more body sensor units and are placed outside or inside of the human body. This sensor gets required data from the human body and sends it wirelessly to the next layer.

- Level 2: Second layer is called the body central unit. The body central unit contains personal servers, and these servers get data from sensors which calculate and manipulate this data, generating the required results. This layer can be based on a wireless computer system, an Android mobile phone, or any GPS supported system that can manipulate received data.

- Level 3: Third layer based on end user's machines where end users are medical assistance or physicians and their machines can be a computer or mobile phones. These machines gather the required information from layer two, and ask end users to provide a response for a patient's fitness. If there are some emergency results, then the machine sends an alarm to the end user and makes it possible to generate a quick response so that patients can get proper treatment.

Lv et al., 2010 studied a health monitoring system. They developed iCare for the senior citizen person. The real-time sensor caters for the daily needs of the elderly persons through reminders, alarms, and medical comprehensive guides, etc. Additionally, the real time sensor makes an active database for further communicating with the family, friends, and doctor when there is a critical situation. Furthermore, it helps to monitor health in a very comprehensive and convenient way.

Kannan et al., 2011 studied the application of real-time sensors for elderly persons and their well being. They developed wearable gloves via which the sensor nodes work, and during an emergency situation, they generate an alarm, short messaging system, and voice call. The sensors measure the heart rate, oxygen content, body temperature, and pressure. This was not developed before; the authors have attempted to make a user-friendly device which can prevent life-threatening conditions.

Baek et al., 2013 investigated real-time sensor applications on body posture in offices, restrooms, homes, etc. It was observed that wrong posture or positioning of the body led to vulnerable outcomes for elderly persons. The authors extensively studied posture and positioning of the body movement via a real-time sensor application. They proposed a device which can be worn as a necklace which includes the sensor nodes and the movement is detected by the sensors. Furthermore, they obtained more than an $80 \%$ sensitive result with $100 \%$ specificity. 
Kantoch et al., 2014 investigated the application of sensors in the health motoring system. They developed a Bluetooth module with wearable sensors. This device monitors the ECG pattern, skin humidity, and body temperature. They calculated the error and it was shown to be around $5 \%$ compared to a certified commercial medical device already available in the market.

Rasyid et al., 2015 investigated the body temperature, heartbeat, and oxygen saturation in blood via real-time sensors. The ubiquitous application of the real time sensor provides a new avenue for the diagnostics industry. Several applications are available, but are still in their infancy, but here in this study, the authors provide a comprehensive approach for the aforesaid parameters. They have developed a Desktop version with printable facilities. The results are in the form of graphs and tables for comprehension. They can be accessed in a specific area network such as from $10 \mathrm{~m}$ to $50 \mathrm{~m}$ via ZigBee without using the wire, which is more appropriate for the WBAN.

Rasyid et al., 2016 investigated a non invasive approach for the detection of the blood glucose level. The invasive approach for the detection of blood glucose is an age old technique which is generally practiced in clinics or hospitals for the diagnosis of diabetes. Of late, the authors have developed a non-invasive approach and quiet reproducible technique via the application of real-time sensors and it plays a pivotal role in the Healthcare industry. The authors have developed glucose sensor monitoring via a Glucometer sensor with an Arduino Uno board and ZigBee module system. Furthermore, in this approach, the patient does not need to be present physically for the routine examination; they can perform the test via a web-based application via the usage of mobile phones.

Wu et al., 2017 investigated the application of sensors based on solar influx gathering. The authors placed an emphasis on evaluating the body temperature distribution, heartbeat, and shelf life of the instrument via web-based smart-phone applications. Additionally, they enhanced the lifetime of the device via several changes in the influx of the solar power. The authors put forward the strategies for resolving the aforementioned tasks.

Table 9. Examples of prototyped applications for WBAN (Wireless Body Area Network). GPRS: General Packet Radio Service; PDA: Personal Digital Assistant.

\begin{tabular}{clcl}
\hline \multicolumn{1}{c}{ References } & \multicolumn{1}{c}{ Parameters } & Sensors & Novelty \\
\hline Lv et al. (2010) [67] & $\begin{array}{l}\text { GSM, General Packet Radio } \\
\text { Service (GPRS), Personal Digital } \\
\text { Assistant (PDA), Bluetooth, } \\
\text { Window Based Smart Phone }\end{array}$ & $\begin{array}{l}\text { Temperature, Humidity, } \\
\text { Moisture, Temperature }\end{array}$ & $\begin{array}{l}\text { Quick Alarm, Regular Reminder, } \\
\text { Medical guidance for elderly }\end{array}$ \\
\hline Kannan et al. (2011) [68] & $\begin{array}{l}\text { Body Temperature, Blood Oxygen, } \\
\text { Heart Rate, BP }\end{array}$ & $\begin{array}{c}\text { Computer, Bluetooth, } \\
\text { GSM }\end{array}$ & $\begin{array}{l}\text { Monitor major parameters of } \\
\text { elderly people }\end{array}$ \\
\hline Baek et al. (2013) [69] & Accelerometer and Gyroscope & ZigBee & $\begin{array}{l}\text { A Fall detection system using } \\
\text { necklace-shaped sensor node }\end{array}$ \\
\hline Kantoch et al. (2014) [70] & $\begin{array}{l}\text { Temperature, Heart Rate, } \\
\text { Skin Humidity }\end{array}$ & $\begin{array}{l}\text { Computer, Bluetooth } \\
\text { living using wearable sensors }\end{array}$ \\
\hline Rasyid et al. (2015) [71] & Rate, Oxygen Saturation in Blood & ZigBee, Computer & $\begin{array}{l}\text { Physiological data can be accessed } \\
\text { wirelessly through ZigBee }\end{array}$ \\
\hline Rasyid et al. (2016) [72] & Glucose Level & Arduino Uno Board, \\
Zigbee & $\begin{array}{l}\text { Deployment of Blood glucose } \\
\text { level monitoring system based } \\
\text { on WBAN }\end{array}$ \\
\hline Wu et al. (2017) [73] & Body Temperature, Heartbeat & Internet of Things & $\begin{array}{l}\text { IoT associated health care } \\
\text { application for WBAN }\end{array}$
\end{tabular}

\subsection{WSN and VANET}

A Vehicular Ad-Hoc Network or VANET may be a variety of the Mobile Ad-Hoc Network or MANET that offers communication between vehicles and roadside base stations. A vehicle in VANET is measured to be an associate intelligent mobile node capable of communication with its neighbors and different vehicles within the network. Digital devices are becoming more and more ubiquitous. The evolution of intelligent components of the digital scheme produces new applications related to security issues that are not resolved. The internet of Vehicles (IoV) consists of vehicles 
that communicate with each other and also with public networks via V2V (vehicle-to-vehicle), V2I (vehicle-to-infrastructure), and V2P (vehicle-to-pedestrian) interactions, which permits the gathering and that is why there is a period of time sharing of critical information regarding the condition of the road network [74]. Figure 8, shows the mechanism of smart vehicle. The Social internet of Things (SIoT) introduces social relationships among objects, making a social network wherever the participants are not humans, but intelligent objects. In this paper, we have incorporated the Social web of Vehicles, a network that permits social interactions among each individual vehicle and drivers. We discuss the technologies and elements of the Social web of Vehicles and its applications. Table 10, depicted the examples of real-time applications for Vehicular Ad Hoc Network (VANET).

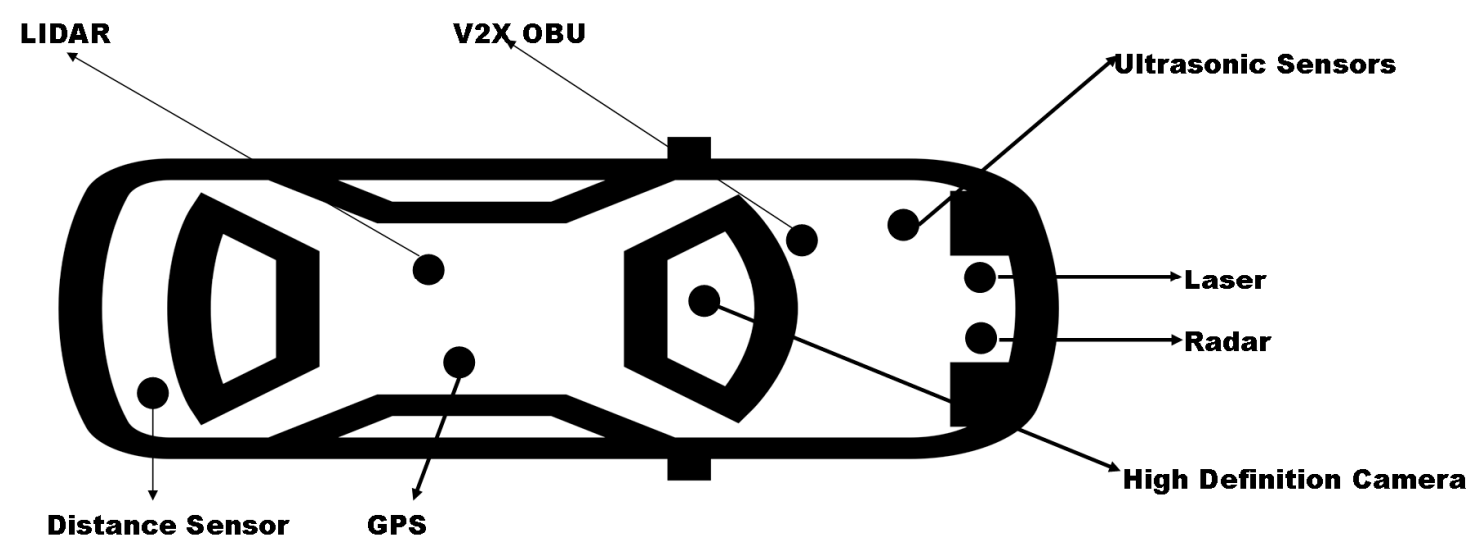

Figure 8. Vehicular Ad Hoc Network (VANET) Smart Vehicle. On-board unit (OBU).

Chatrapathi et al., 2015 investigated the application of sensors in the road traffic system. The authors have extensively worked on Internet of Things (IoT) and VANET. The combinatorial approach of the aforementioned framework has been employed to detect accidents, avoid secondary accidents, and contact the ambulance in a timely manner. Additionally, the sensor will communicate with the ambulances and hospital for further correspondence regarding the incident. The authors inferred that their approach reduces the time for contacting the aforesaid, which might lead to a chance of saving a life.

Tai el al., 2017 developed a novel traffic monitoring system for VANET-based applications. Their proposed model is able to generate and analyze traffic condition reports, including the current time and location of the traffic with the help of image processing techniques. This approach exhibited around $85 \%$ accuracy in maintaining the authenticity of the data, $84.093 \mathrm{~m} / \mathrm{s}$ for the implementation time, and a $0.011 \%$ increase in computation overhead applying for the confidentiality scheme.

Table 10. Examples of prototyped applications for VANET.AMS: Accident Management System; STMS: Secure Traffic Monitoring System; VPID: Virtual Piconet ID.

\begin{tabular}{cccc}
\hline References & Application & Sensors & City Deployed in \\
\hline Chatrapathi et al. (2015) [75] & $\begin{array}{c}\text { Accident Management } \\
\text { System (AMS) }\end{array}$ & $\begin{array}{c}\text { Mechanical sensors, } \\
\text { Biometric Sensors }\end{array}$ & Coimbatore, India \\
\hline Taie et al. (2017) [76] & $\begin{array}{c}\text { Secure Traffic Monitoring } \\
\text { System (STMS) }\end{array}$ & $\begin{array}{c}\text { GPS, Virtual Piconet ID } \\
\text { (VPID) }\end{array}$ & Cairo, Egypt \\
\hline
\end{tabular}

WSN and Automated Vehicles

Automatic vehicles possess the attribute of safety critical management functions such as directing, regulating, or the usage of a brake. The aforesaid attributes will work whenever a manual driver input is unavailable. Automatic vehicles could be "autonomous" (i.e., solely based on vehicle sensors) or could use a communications system to connect with the vehicle technology. This communication 
will work wirelessly with the ground level lane during the movement of the vehicle. The automatic vehicles utilize multiple techniques to comprehend their ambiance such as a microwave radar, optical device light, GPS, odometer, and PC version. The advanced mechanism interprets the sensory information to recognize the appropriate course-plotting ways, in addition to hindrances and important accumulations. Furthermore, the autonomous car possesses a mechanical system which is capable of investigating the sensory information and is able to discriminate the other vehicle/car which is moving in the same lane with the same destination path specified. Additionally, the input parameter such as connectivity plays a vital role and it is recognized as an advantage [77]. In order to prevent the traffic accidents, automatic vehicles could a good choice. The automatic vehicle showed some promising merits such as a reduction in fuel consumption, help with parking in busy parking areas, a reduction in the crime rate of the city, as well as being able to cater for youth, senior, or elderly persons and physically challenged persons. For commercialization and awareness among society regarding the automatic vehicle, business tycoons should take the initiative and be facilitated for their business models. Table 11, represent the examples of prototyped applications for automated vehicles.

Dagher et al., 2014 investigated the smart navigating system. Due to rapid industrialization and urban development, the number of vehicles has been increased in a very rapid manner. To avoid congestion in the streets and roads, the parking problem, which is increasing in everyday life, needs to be addressed. To resolve this issue, the authors implemented real-time sensors in the smart cities to eradicate the problems for better daily life. The authors have developed a model which is coined as Angle of Arrival (AoA), which depicts and measures the vehicle position on the map. Additionally, the authors have simulated the result and the obtained data gave a significant outcome, enhancing the positioning of the vehicle.

Srinivasan et al., 2016 investigated the application of real time sensors with the help of IoT and sensor networks. They applied these approaches to road safety, avoiding traffic regulation violation.

Table 11. Examples of prototyped applications for automated vehicles.

\begin{tabular}{cccc}
\hline References & Application & Motes & City Deployed in \\
\hline Dagher et al. (2014) [78] & UNS-Ubiquitous Navigation System & RF based, Google Maps & Lille, France \\
\hline Srinivasan et al. (2016) [79] & FWI-Fire Weather Index & Internet of Things, GPS Sensors & Oman \\
\hline
\end{tabular}

\subsection{WSN and Military}

The United States military used WSN for the first time in a military application in 1960, called the Sound Surveillance System (SOSUS). This is used for the detection and tracking of submarines. Nowadays, WSN is used in many ways like tracking or the moving of a solider or vehicles during the surveillance mission. This is also used for collecting data or necessary information from the target area and sending it to the base station. However, the military needs to improve the quality of WSN security by the routing algorithm [80]. Table 12, represent the examples of real-time applications for Military.

Arora et al., 2005 investigated the deployment of a dense wireless network sensor, which is coined as "Tripwire". The reason behind this project is to limit the intruder from crossing the border, including any kind of sabotages, and from smuggling important plant species which might be an indigenous species and will be a national treasure for the country. Overall, for security reasons, the sensors need to be deployed in the border region between the countries.

Acar et al., 2006 investigated the underwater sensor network for monitoring fish detection, collision avoidance, underwater vehicles, and long-term ecological inspection. The authors studied how to enhance the shelf life of the sensor and increment of the bandwidth and obtained a comprehensive result. 
Table 12. Examples of prototyped applications for the military.

\begin{tabular}{cccc}
\hline References & Application & Sensors & City Deployed in \\
\hline Arora et al. (2005) [81] & ExScal & $\begin{array}{c}\text { Magnetometer, Acoustics, } \\
\text { Passive Infrared Sensor (PIR) }\end{array}$ & Florida, USA \\
\hline Acar et al. (2006) [82] & ACMENet & Underwater Acoustic Sensors & European Union \\
\hline
\end{tabular}

\section{SWOT (Strength, Weakness, Opportunities, and Threat) Analysis of WSNs}

The acronym of SWOT is Strength, Weakness, Opportunities, and Threat. SWOT is applied in this study to diagnose the merits and demerits of the real time application of the WSN. A WSN is ubiquitously applied in every aspect of daily life for its convenience in terms of intelligence and wireless networking such as smart and improved transportation systems. An attempt has been made in this paper to recognize the SWOT analysis for the real time application of the WSN. Furthermore, this kind of approach has not yet been performed by any researchers of this field. We have incorporated the analysis of SWOT in this study. Figure 9, presents an illustration of SWOT depicting the explanations.

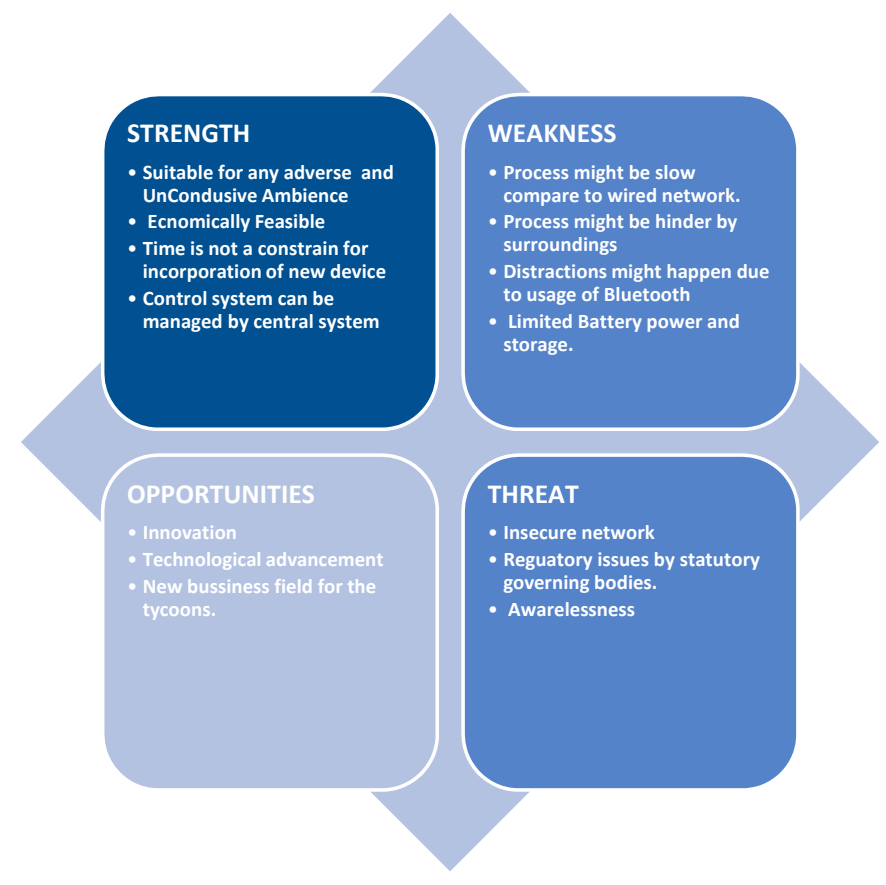

Figure 9. SWOT (Strength, Weakness, Opportunities, and Threat) Analysis of WSNs.

\section{Conclusions}

In this study, we have copiously reviewed the usage of the WSN in diverse fields. Due to the rapid and progressive development of society, continuous innovation is an utmost requirement for human civilization and its development. It has been observed that via the application of this intelligent and smart technology, quality of life has been enriched. WSN has the potential to be employed in the measurement and controlling applications. With the aid of WSN, the incrementing spaces could be controlled and observed long-term data could be transferred at that scale, which is hard to achieve. Without any external guideline and supervision, it has the ability to develop a network itself. WSN is beneficial for numerous daily life applications where we have tried to demonstrate through the various usage of WSNs. The scope of WSN applications is not limited to, environmental monitoring, the surveillance of criminal activities, healthcare, and infrastructure monitoring. Each application has its own features and requirements. However, there are still several challenges that need to be addressed in order to augment the development of WSN applications such as power management, 
security, and communication protocols. By addressing these issues, the application of WSN can be helpful in numerous fields like predictive maintenance, high confidence-asset tracking, facilitating new knowledge, wind speed and direction, improving food, seismic detection, mechanical stress detection after earthquakes, intelligent buildings, improving productivity, and energy saving smart grids. We have tried our best to categorize each application scenario specifically and have included prototype examples that adequately fit the scenario. This is accomplished by investigating the extensive literature review of various research papers and implication contributions to this area. Several technologists and researchers envisage that the sensor network will bring several fields together in order to broaden its horizon. Furthermore, the incorporation of additional advancement in the real-time applications of WSN possesses an extensive range of scope in this emerging research field.

Acknowledgments: The authors thankfully acknowledge the Hebei University of Technology for providing all the necessary materials pertaining to the completion of this paper. Ahmad Ali thankfully acknowledged the China Scholarship Council (CSC) for providing the Scholarship towards his Doctoral study from Hebei University of Technology, Tianjin, China.

Author Contributions: Ahmad Ali collected the data and designed the review model. Yu Ming supervises and mentored the work. Sagnik Chakraborty, Ahmad Ali and Saima Iram jointly discussed, analyzed, and wrote the paper. Additionally, Sagnik Chakraborty reviewed the whole work and provided suggestions for the enrichment of the paper.

Conflicts of Interest: The authors declare no conflicts of interest.

\section{References}

1. Akyildiz, I.; Su, W.; Sankarasubramaniam, Y.; Cayirci, E. Wireless sensor networks: A survey. Comput. Netw. 2002, 38, 393-422. [CrossRef]

2. Zhou, Y.; Fang, Y.; Zhang, Y. Securing wireless sensor networks: A survey. IEEE Commun. Surv. Tutor. 2008, 10, 6-28. [CrossRef]

3. Diamond, S.; Ceruti, M. Application of Wireless Sensor Network to Military Information Integration. In Proceedings of the 2007 5th IEEE International Conference on Industrial Informatics, Vienna, Austria, 23-27 July 2007.

4. Alemdar, H.; Ersoy, C. Wireless sensor networks for healthcare: A survey. Comput. Netw. 2010, 54, 2688-2710. [CrossRef]

5. Bal, M. Industrial applications of collaborative Wireless Sensor Networks: A survey. In Proceedings of the 2014 IEEE 23rd International Symposium on Industrial Electronics (ISIE), Istanbul, Turkey, 1-4 June 2014.

6. Chee-Yee, C.; Kumar, S. Sensor networks: Evolution, opportunities, and challenges. Proc. IEEE 2003, 91, 1247-1256. [CrossRef]

7. Barroca, N.; Borges, L.; Velez, F; Monteiro, F; Górski, M.; Castro-Gomes, J. Wireless sensor networks for temperature and humidity monitoring within concrete structures. Constr. Build. Mater. 2013, 40, 1156-1166. [CrossRef]

8. Li, M.; Lin, H. Design and Implementation of Smart Home Control Systems Based on Wireless Sensor Networks and Power Line Communications. IEEE Trans. Ind. Electron. 2015, 62, 4430-4442. [CrossRef]

9. Sandhu, M.; Javaid, N.; Jamil, M.; Khan, Z.; Imran, M.; Ilahi, M.; Khan, M. Modeling mobility and psychological stress based human postural changes in wireless body area networks. Comput. Hum. Behav. 2015, 51, 1042-1053. [CrossRef]

10. Basagni, S.; Carosi, A.; Petrioli, C.; Phillips, C. Coordinated and controlled mobility of multiple sinks for maximizing the lifetime of wireless sensor networks. Wirel. Netw. 2011, 17, 759-778. [CrossRef]

11. Honjo, T.; Yamato, H.; Mikami, T.; Grimmond, C. Network optimization for enhanced resilience of urban heat island measurements. Sustain. Cities Soc. 2015, 19, 319-330. [CrossRef]

12. Yu, T.; Lin, C.; Chen, C.; Lee, W.; Lee, R.; Tseng, C.; Liu, S. Wireless sensor networks for indoor air quality monitoring. Med. Eng. Phys. 2013, 35, 231-235. [CrossRef] [PubMed]

13. Gkikopouli, A.; Nikolakopoulos, G.; Manesis, S. A survey on Underwater Wireless Sensor Networks and applications. In Proceedings of the 2012 20th Mediterranean Conference on Control \& Automation (MED), Barcelona, Spain, 3-6 July 2012. 
14. Nadeem, F.; Leitgeb, E.; Awan, M.; Chessa, S. Comparing the Life Time of Terrestrial Wireless Sensor Networks by Employing Hybrid FSO/RF and Only RF Access Networks. In Proceedings of the 2009 Fifth International Conference on Wireless and Mobile Communications, Cannes, France, 23-29 August 2009.

15. AL-Kadi, T.; AL-Tuwaijri, Z.; AL-Omran, A. Wireless Sensor Networks for Leakage Detection in Underground Pipelines: A Survey Paper. Procedia Comput. Sci. 2013, 21, 491-498. [CrossRef]

16. Sun, Z.; Akyildiz, I.; Hancke, G. Dynamic Connectivity in Wireless Underground Sensor Networks. IEEE Trans. Wirel. Commun. 2011, 10, 4334-4344. [CrossRef]

17. Zaman, I.; Gellhaar, M.; Dede, J.; Koehler, H.; Foerster, A. Demo: Design and Evaluation of MoleNet for Wireless Underground Sensor Networks. In Proceedings of the 2016 IEEE 41st Conference on Local Computer Networks Workshops (LCN Workshops), Dubai, UAE, 7-10 November 2016.

18. Ayaz, M.; Baig, I.; Abdullah, A.; Faye, I. A survey on routing techniques in underwater wireless sensor networks. J. Netw. Comput. Appl. 2011, 34, 1908-1927. [CrossRef]

19. Anguita, D.; Brizzolara, D.; Parodi, G. Building an Underwater Wireless Sensor Network Based on Optical: Communication: Research Challenges and Current Results. In Proceedings of the 2009 Third International Conference on Sensor Technologies and Applications, Athens/Glyfada, Greece, 18-23 June 2009.

20. Goyal, N.; Dave, M.; Verma, A. Data aggregation in underwater wireless sensor network: Recent approaches and issues. J. King Saud Univ. Comput. Inf. Sci. 2017. [CrossRef]

21. Tonneau, A.; Mitton, N.; Vandaele, J. A Survey on (mobile) Wireless Sensor Network Experimentation Testbeds. In Proceedings of the 2014 IEEE International Conference on Distributed Computing in Sensor Systems, Marina Del Rey, CA, USA, 26-28 May 2014.

22. Cho, J.; Swami, A.; Chen, I. A Survey on Trust Management for Mobile Ad Hoc Networks. IEEE Commun. Surv. Tutor. 2011, 13, 562-583. [CrossRef]

23. Shen, H.; Bai, G. Routing in wireless multimedia sensor networks: A survey and challenges ahead. J. Netw. Comput. Appl. 2016, 71, 30-49. [CrossRef]

24. Akyildiz, I.; Melodia, T.; Chowdhury, K. A survey on wireless multimedia sensor networks. Comput. Netw. 2007, 51, 921-960. [CrossRef]

25. Hayat, M.; Khan, H.; Iqbal, Z.; Ur Rahman, Z.; Tahir, M. Multimedia sensor networks: Recent trends, research challenges and future directions. In Proceedings of the 2017 International Conference on Communication, Computing and Digital Systems (C-CODE), Islamabad, Pakistan, 8-9 March 2017.

26. Othman, M.; Shazali, K. Wireless Sensor Network Applications: A Study in Environment Monitoring System. Procedia Eng. 2012, 41, 1204-1210. [CrossRef]

27. Boustani, A.; Girod, L.; Offenhuber, D.; Britter, R.; Wolf, M.; Lee, D.; Miles, S.; Biderman, A.; Ratti, C. Investigation of the waste-removal chain through pervasive computing. IBM J. Res. Dev. 2011, 55, 11:1-11:11. [CrossRef]

28. Mao, X.; Miao, X.; He, Y.; Li, X.; Liu, Y. CitySee: Urban $\mathrm{CO}_{2}$ monitoring with sensors. In Proceedings of the 2012 IEEE INFOCOM, Orlando, FL, USA, 25-30 March 2012.

29. Hasenfratz, D.; Saukh, O.; Walser, C.; Hueglin, C.; Fierz, M.; Arn, T.; Beutel, J.; Thiele, L. Deriving high-resolution urban air pollution maps using mobile sensor nodes. Pervasive Mob. Comput. 2015, 16, 268-285. [CrossRef]

30. Jiang, Q.; Kresin, F.; Bregt, A.; Kooistra, L.; Pareschi, E.; van Putten, E.; Volten, H.; Wesseling, J. Citizen Sensing for Improved Urban Environmental Monitoring. J. Sens. 2016, 2016. [CrossRef]

31. Ahonen, T.; Virrankoski, R.; Elmusrati, M. Greenhouse Monitoring with Wireless Sensor Network. In Proceedings of the 2008 IEEE/ASME International Conference on Mechtronic and Embedded Systems and Applications, Beijing, China, 12-15 October 2008.

32. Farm Management. Available online: http://www.farmmanagement.pro/the-use-of-wireless-sensornetworks-in-precision-agriculture (accessed on 15 September 2017).

33. Pahuja, R.; Verma, H.; Uddin, M. A Wireless Sensor Network for Greenhouse Climate Control. IEEE Pervasive Comput. 2013, 12, 49-58. [CrossRef]

34. Liu, J. Design and Implementation of an Intelligent Environmental-Control System: Perception, Network, and Application with Fused Data Collected from Multiple Sensors in a Greenhouse at Jiangsu, China. Int. J. Distrib. Sens. Netw. 2016, 12, 5056460. [CrossRef]

35. Queiroz, D.; Alencar, M.; Gomes, R.; Fonseca, I.; Benavente-Peces, C. Survey and systematic mapping of industrial Wireless Sensor Networks. J. Netw. Comput. Appl. 2017, 97, 96-125. [CrossRef] 
36. Ni, D.; Chin, C. Sensor Network for Gas Meter Application. In Proceedings of the 2009 Third International Conference on Sensor Technologies and Applications, Athens/Glyfada, Greece, 18-23 June 2009.

37. Chen, C.; Lee, D. Energy Saving Effects of Wireless Sensor Networks: A Case Study of Convenience Stores in Taiwan. Sensors 2011, 11, 2013-2034. [CrossRef] [PubMed]

38. Valverde, J.; Rosello, V.; Mujica, G.; Portilla, J.; Uriarte, A.; Riesgo, T. Wireless Sensor Network for Environmental Monitoring: Application in a Coffee Factory. Int. J. Distrib. Sens. Netw. 2012, 8, 638067. [CrossRef]

39. Schirrmann, M.; Gebbers, R.; Kramer, E.; Seidel, J. Soil pH Mapping with an On-The-Go Sensor. Sensors 2011, 11, 573-598. [CrossRef] [PubMed]

40. Neethirajan, S. Recent advances in wearable sensors for animal health management. Sens. Bio-Sens. Res. 2017, 12, 15-29. [CrossRef]

41. Wark, T.; Corke, P.; Sikka, P.; Klingbeil, L.; Guo, Y.; Crossman, C.; Valencia, P.; Swain, D.; Bishop-Hurley, G. Transforming Agriculture through Pervasive Wireless Sensor Networks. IEEE Pervasive Comput. 2007, 6, 50-57. [CrossRef]

42. Mashoko Nkwari, P.; Rimer, S.; Paul, B. Cattle monitoring system using wireless sensor network in order to prevent cattle rustling. In Proceedings of the 2014 IST-Africa Conference, Le Meridien Ile Maurice, Mauritius, 7-9 May 2014.

43. Wamuyu, P. A Conceptual Framework for Implementing a WSN Based Cattle Recovery System in Case of Cattle Rustling in Kenya. Technologies 2017, 5, 54. [CrossRef]

44. Dessart, N.; Hunel, P. Data collection using WSN for counting individuals and habitat characterization. J. Comput. Sci. 2014, 5, 624-632. [CrossRef]

45. Stattner, E.; Vidot, N.; Hunel, P.; Collard, M. Wireless sensor network for habitat monitoring: A counting heuristic. In Proceedings of the 37th Annual IEEE Conference on Local Computer Networks-Workshops, Clearwater Beach, FL, USA, 22-25 October 2012.

46. Mainwaring, A.; Culler, D.; Polastre, J.; Szewczyk, R.; Anderson, J. Wireless sensor networks for habitat monitoring. In Proceedings of the 1st ACM International Workshop on Wireless Sensor Networks and Applications-WSNA'02, Atlanta, GA, USA, 28 September 2002.

47. Naumowicz, T.; Freeman, R.; Kirk, H.; Dean, B.; Calsyn, M.; Liers, A.; Braendle, A.; Guilford, T.; Schiller, J. Wireless Sensor Network for habitat monitoring on Skomer Island. In Proceedings of the 2010 IEEE 35th Conference onLocal Computer Networks (LCN), Denver, CO, USA, 10-14 October 2010.

48. Jan, M.; Nanda, P.; He, X.; Liu, R. A Sybil attack detection scheme for a forest wildfire monitoring application. Future Gener. Comput. Syst. 2016. [CrossRef]

49. Gaikwad, S.; Patil, R.; Khandare, A.; Rai, A. Design WSN node for protection of forest trees against poaching based on ZigBee. In Proceedings of the 2015 IEEE International Conference on Electronics, Computing and Communication Technologies (CONECCT), Bangalore, India, 10-11 July 2015.

50. The Dynamics of Forest Canopy Motion. Available online: http://today.uconn.edu/2010/01/the-dynamicsof-forest-canopy-motion / (accessed on 15 September 2017).

51. Son, B.; Her, Y.; Kim, J. A Design and Implementation of Forest-Fires Surveillance System based on Wireless Sensor Network for South Korea Mountains. Int. J. Comput. Sci. Netw. Secur. 2006, 6, 9B.

52. Hefeeda, M.; Bagheri, M. Wireless Sensor Networks for Early Detection of Forest Fires. In Proceedings of the 2007 IEEE Internatonal Conference on Mobile Adhoc and Sensor Systems, Pisa, Italy, 8-11 October 2007.

53. Kim, D.; Cano, J.; Wang, W.; De Rango, F.; Hua, K. Underwater Wireless Sensor Networks 2015. Int. J. Distrib. Sens. Netw. 2015, 11, 623042. [CrossRef]

54. Zenia, N.; Aseeri, M.; Ahmed, M.; Chowdhury, Z.; Shamim Kaiser, M. Energy-efficiency and reliability in MAC and routing protocols for underwater wireless sensor network: A survey. J. Netw. Comput. Appl. 2016, 71, 72-85. [CrossRef]

55. Ilyas, N.; Akbar, M.; Ullah, R.; Khalid, M.; Arif, A.; Hafeez, A.; Qasim, U.; Khan, Z.; Javaid, N. SEDG: Scalable and Efficient Data Gathering Routing Protocol for Underwater WSNs. Procedia Comput. Sci. 2015, 52, 584-591. [CrossRef]

56. Water Quality Monitoring in Europe's Largest Fluvial Aquarium. Available online: http:/ /www.libelium.com/ water-quality-monitoring-europe-largest-fluvial-aquarium-zaragoza/ (accessed on 15 November 2017). 
57. Gray, A.; Sadler, J.; Kit, O.; Kyzirakos, K.; Karpathiotakis, M.; Calbimonte, J.; Page, K.; García-Castro, R.; Frazer, A.; Galpin, I.; et al. A Semantic Sensor Web for Environmental Decision Support Applications. Sensors 2011, 11, 8855-8887. [CrossRef] [PubMed]

58. Whittle, A.J.; Allen, M.; Preis, A.; Iqbal, M. Sensor Networks for Monitoring and Control of Water Distribution Systems. In Proceedings of the 6th International Conference on Structural Health Monitoring of Intelligent Infrastructure (SHMII), Hong Kong, China, 9-11 December 2013.

59. Kim, H.; Cho, H. SOUNET: Self-Organized Underwater Wireless Sensor Network. Sensors 2017, 17, 283. [CrossRef] [PubMed]

60. Milenković, A.; Otto, C.; Jovanov, E. Wireless sensor networks for personal health monitoring: Issues and an implementation. Comput. Commun. 2006, 29, 2521-2533. [CrossRef]

61. Ko, J.; Gao, T.; Terzis, A. Empirical study of a medical sensor application in an urban emergency department. In Proceedings of the 4th International ICST Conference on Body Area Networks, Los Angeles, CA, USA, 1-3 April 2009.

62. Hii, P.; Chung, W. A Comprehensive Ubiquitous Healthcare Solution on an Android ${ }^{\mathrm{TM}}$ Mobile Device. Sensors 2011, 11, 6799-6815. [CrossRef] [PubMed]

63. Kakria, P.; Tripathi, N.; Kitipawang, P. A Real-Time Health Monitoring System for Remote Cardiac Patients Using Smartphone and Wearable Sensors. Int. J. Telemed. Appl. 2015, 2015, 1-11. [CrossRef] [PubMed]

64. Yan, S.; Ma, H.; Li, P.; Song, G.; Wu, J. Development and Application of a Structural Health Monitoring System Based on Wireless Smart Aggregates. Sensors 2017, 17, 1641. [CrossRef] [PubMed]

65. Yuce, M. Implementation of wireless body area networks for healthcare systems. Sens. Actuators A Phys. 2010, 162, 116-129. [CrossRef]

66. Salehi, S.; Razzaque, M.; Tomeo-Reyes, I.; Hussain, N. IEEE 802.15.6 standard in wireless body area networks from a healthcare point of view. In Proceedings of the 2016 22nd Asia-Pacific Conference on Communications (APCC), Yogyakarta, Indonesia, 25-27 August 2016.

67. Lv, Z.; Xia, F.; Wu, G.; Yao, L.; Chen, Z. iCare: A Mobile Health Monitoring System for the Elderly. In Proceedings of the 2010 IEEE/ACM Int'l Conference on Green Computing and Communications \& Int'l Conference on Cyber, Physical and Social Computing, Hangzhou, China, 18-20 December 2010.

68. Megalingam, R.; Radhakrishnan, V.; Jacob, D.; Unnikrishnan, D.; Sudhakaran, A. Assistive Technology for Elders: Wireless Intelligent Healthcare Gadget. In Proceedings of the 2011 IEEE Global Humanitarian Technology Conference, Seattle, WA, USA, 30 October-1 November 2011.

69. Woon-Sung, B.; Dong-Min, K.; Bashir, F.; Pyun, J.Y. Real life applicable fall detection system based on wireless body area network. In Proceedings of the 2013 IEEE 10th Consumer Communications and Networking Conference (CCNC), Las Vegas, NV, USA, 11-14 January 2013.

70. Kantoch, E.; Augustyniak, P.; Markiewicz, M.; Prusak, D. Monitoring activities of daily living based on wearable wireless body sensor network. In Proceedings of the 2014 36th Annual International Conference of the IEEE Engineering in Medicine and Biology Society, Chicago, IL, USA, 26-30 August 2014.

71. Al Rasyid, M.; Lee, B.; Sudarsono, A. Wireless body area network for monitoring body temperature, heart beat and oxygen in blood. In Proceedings of the 2015 International Seminar on Intelligent Technology and Its Applications (ISITIA), Surabaya, Indonesia, 20-21 May 2015.

72. Rasyid, M.; Saputra, F.; Christian, A. Implementation of blood glucose levels monitoring system based on Wireless Body Area Network. In Proceedings of the 2016 IEEE International Conference on Consumer Electronics-Taiwan (ICCE-TW), Nantou, Taiwan, 27-29 May 2016.

73. Wu, T.; Wu, F.; Redoute, J.; Yuce, M. An Autonomous Wireless Body Area Network Implementation Towards IoT Connected Healthcare Applications. IEEE Access 2017, 5, 11413-11422. [CrossRef]

74. Tariq, S.; Hyunsoo, C.; Wasiq, C. Heemin Park Controlled parking for self-driving cars. In Proceedings of the 2016 IEEE International Conference on Systems, Man, and Cybernetics (SMC), Budapest, Hungary, 9-12 October 2016.

75. Chatrapathi, C.; Rajkumar, M.; Venkatesakumar, V. VANET based integrated framework for smart accident management system. In Proceedings of the 2015 International Conference on Soft-Computing and Networks Security (ICSNS), Coimbatore, India, 25-27 February 2015.

76. Taie, S.; Taha, S. A novel secured traffic monitoring system for VANET. In Proceedings of the 2017 IEEE International Conference on Pervasive Computing and Communications Workshops (PerCom Workshops), Kailua-Kona, HI, USA, 13-17 March 2017. 
77. You, Z.; Chen, S.; Wang, Y. Novel dynamic data aggregation scheme for WSN based intelligent vehicle systems. Intell. Decis. Technol. 2016, 10, 105-114. [CrossRef]

78. Dagher, R.; Mitton, N.; Amadou, I. Towards WSN-aided navigation for vehicles in smart cities: An application case study. In Proceedings of the 2014 IEEE International Conference on Pervasive Computing and Communication Workshops (PERCOM WORKSHOPS), Budapest, Hungary, 24-28 March 2014.

79. Srinivasan, R.; Sharmili, A.; Saravanan, S.; Jayaprakash, D. Smart vehicles with everything. In Proceedings of the 2016 2nd International Conference on Contemporary Computing and Informatics (IC3I), Greater Noida, India, 14-17 December 2016.

80. Winkler, M.; Street, M.; Tuchs, K.; Wrona, K. Wireless Sensor Networks for Military Purposes. In Autonomous Sensor Network; Springer: Berlin/Heidelberg, Germany, 2012; pp. 365-394.

81. Açar, G.; Adams, A. ACMENet: An underwater acoustic sensor network protocol for real-time environmental monitoring in coastal areas. IEE Radar Sonar Navig. 2006, 153, 365. [CrossRef]

82. Arora, A.; Ramnath, R.; Ertin, E.; Sinha, P.; Bapat, S.; Naik, V.; Kulathumani, V.; Zhang, H.; Cao, H.; Sridharan, M.; et al. ExScal: Elements of an Extreme Scale Wireless Sensor Network. In Proceedings of the 11th IEEE International Conference on Embedded and Real-Time Computing Systems and Applications (RTCSA`05), Hong Kong, China, 17-19 August 2005.

(c) 2017 by the authors. Licensee MDPI, Basel, Switzerland. This article is an open access article distributed under the terms and conditions of the Creative Commons Attribution (CC BY) license (http://creativecommons.org/licenses/by/4.0/). 